\section{Rheumatoid arthritis: an antigenic chameleon}

\author{
Eugen Feist, ${ }^{1}$ Guenter Steiner ${ }^{2}$
}

Nowadays, rheumatologists and immunologists have to be absorptive like a sponge -from a breathtaking progress in the basic research field to emerging approaches in diagnosis and treatment of complex disorders like rheumatoid arthritis (RA), there is always exciting and sometimes unexpected news to digest.

For those who are particularly interested in the autoimmune response in RA, we have to realise that we are engaged with a true chameleon. Since the discovery more than 15 years ago of humoral autoimmune reactivities to epitopes containing the unusual amino acid citrulline, ${ }^{1}$ many new autoimmune targets have been discovered and, even more important, also different post-translational modifications to push their antigenic properties. Meanwhile the best understood process represents enzymatic deimination of peptide-or protein-bound arginine to citrulline. In genetically prone individuals, this activity creates highly specific recognition motives for auto-antibodies (anti-citrullinated protein/peptide antibodies, ACPAs), which serve as excellent diagnostic markers, especially in the early phases of disease. Since citrullination, as a physiological process, is also triggered by smoking, it is tempting to assume that there might be a connection. In fact, carriers of distinct HLA alleles coding for the so called shared epitope (particularly HLA-DR*0401) have the highest risk for the development of ACPA, and subsequently RA, especially when they are smokers. But is this the whole story how smoking contributes to the risk for RA?

With regard to smoking and posttranslational modification of proteins, another process, named carbamylation, could also be of interest. In contrast to citrullination, which is performed enzymatically by peptidylarginine deiminases (PAD), carbamylation is another

\footnotetext{
${ }^{1}$ Department of Rheumatology and Clinical Immunology, Charite University Hospital, Berlin, Germany; ' ${ }^{2}$ Department of Rheumatology, Clinic for Internal Medicine III, Medical University Vienna, Vienna, Austria
}

Correspondence to Dr Eugen Feist, Department of Rheumatology and Clinical Immunology, Charite University Hospital, Charitéplatz 1, Berlin 10117, Germany; eugen.feist@charite.de non-enzymatic form of protein modification inducible by urea, among others, but also by compounds contained in tobacco smoke, namely cyanides. Basically, a homologous citrulline structure (homocitrulline) is created from lysine by extension of a single carbon residue. The burning question is of course, is this somehow linked to the development of arthritis?

First, strong evidence that this could be the case in an animal model has been published; immunisation of mice with homocitrulline-containing peptides induced chemotaxis, $\mathrm{T}$ cell activation and antibody production. $^{2}$ Of note, intra-articular injection of peptides containing homocitrulline or citrulline caused erosive arthritis in mice which had been immunised with homocitrulline-containing peptides. The most pronounced form of arthritis was observed in mice that had received intra-articular citrullinated peptides, suggesting a potential cross-reactivity between both antigens. Importantly, the occurrence of autoantibodies against carbamylated antigens was recently shown in patients with established RA, and subsequently even in individuals prior to diagnosis of RA. Although these studies demonstrated a considerable but not complete overlap between the different antibody specificities against either citrulline or homocitrulline containing antigens, this was only partly due to cross-reactivity. ${ }^{3} 4$ These findings suggest that carbamylation in addition to citrullination could represent another crucial process in the pathogenesis of RA. Now what about smoking?

In the article by Jiang et al, ${ }^{5}$ this relationship was investigated in two representative cohorts of patients with RA for whom genetic background information was available. First, the results provided further evidence that the antibody responses against carbamylated antigens (anti-CarP) are distinct from the formation of ACPA (in this case determined by the anti-CCP assay which detects most ACPA subspecificities). In fact, a considerable proportion (approx. 35-45\%) of RA patients expressed antibodies against carbamylated antigens including carbamylated fibrinogen, of whom only very few (4-7\%) were ACPA negative. In agreement with this observation, it was even more remarkable that the genetic background and smoking behaviour were different between patients with ACPA compared to anti-CarP reactivities. Although, as expected, the known risk factors for ACPA development were confirmed, patients with singular antibody reactivity against carbamylated antigens showed a different genetic background (ie, no association with the shared epitope), and even more surprisingly, no strong association with smoking status. As the authors acknowledged, the study was clearly limited by the fact that the remaining group of ACPA-/CarP+ patients was rather small, which does of course influence the statistical analysis. Furthermore, it cannot be fully excluded that the anti-CarP immune memory is less stable compared to ACPA and that the shared epitope perpetuates the formation of ACPA, especially in smokers. Further studies are therefore required to characterise these immune responses in more detail. The relevant targets still need to be identified because the data generated so far mainly rely on an assay employing carbamylated fetal calf serum, even though in the current study carbamylated fibrinogen was used as additional antigen. In particular, it needs to be clarified whether antibodies against other defined antigens potentially modified by carbamylation, such as vimentin or $\alpha$-enolase, are detectable in patients with RA, and whether these antibodies are differently induced during certain stages of disease and/or are characteristic markers for a specific subset of RA.

Contributors Both authors have contributed significantly the editorial and have read and approved the manuscript.

\section{Competing interests None.}

Provenance and peer review Commissioned; externally peer reviewed.

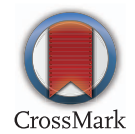

To cite Feist E, Steiner G. Ann Rheum Dis 2014;73:1753-1754.

Received 23 April 2014

Revised 22 May 2014

Accepted 25 May 2014

Published Online First 6 June 2014

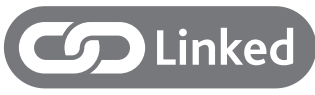

- http://dx.doi.org/10.1136/annrheumdis-2013205109

Ann Rheum Dis 2014;73:1753-1754.

doi:10.1136/annrheumdis-2014-205307 


\section{REFERENCES}

1 Schellekens GA, de Jong BA, van den Hoogen FH, et al. Citrulline is an essential constituent of antigenic determinants recognized by rheumatoid arthritis-specific autoantibodies. J Clin Invest 1998;101:273-81.

2 Mydel P, Wang Z, Brisslert M, et al. Carbamylation-dependent activation of $T$ cells: a novel mechanism in the pathogenesis of autoimmune arthritis. J Immunol 2010;184:6882-90.

3 Shi J, Knevel R, Suwannalai P, et al. Autoantibodies recognizing carbamylated proteins are present in sera of patients with rheumatoid arthritis and predict joint damage. Proc Natl Acad Sci USA 2011;108:17372-7.

4 Shi J, van de Stadt LA, Levarht EW, et al. Anti-carbamylated protein (anti-CarP) antibodies precede the onset of rheumatoid arthritis. Ann Rheum Dis 2014;73:780-3.

5 Jiang $X$, Trouw LA, van Wesemael TJ, et al. Anti-CarP antibodies in two large cohorts of patients with rheumatoid arthritis and their relationship to genetic risk factors, cigarette smoking and other autoantibodies. Ann Rheum Dis 2014;73:1761-8. 